distal interphalangeal (DIP) joints, inflammatory involvement of both flexor (tenosynovitis) and extensor (paratenonitis) tendons, nail bed vascularization, synovio-entheseal complex at DIP level, enthesitis of flexor tendon, and bone proliferation. Grey-scale (GS) and power-Doppler (PD) synovitis and tenosynovitis were assessed according to OMERACT scores (0-3). Nail bed vascularization was scored 0 to 3 . The other US lesions were scored 0 (absent) or 1 (present), both in GS and PD. The standard (HAQ) questionnaire, tender and swollen joint count, patient-reported tenderness, global disease activity scored by physician and by patient were assessed in all patients.

Results: Twelve (60\%) patients presented hand and $8(40 \%)$ patients foot dactylitis. Twelve $(60 \%)$ patients had single dactylitis. Eight patients presented with multiple dactylitis: 4 dactylitis in 1 patient, $(5 \%), 3$ dactylitis in 4 patients $(20 \%)$ patients and 2 dactylitis in $3(15 \%)$ patients. Soft tissue thickening was present in all 34 dactylitis. MCP/MTP joint synovitis was present in 28 digits (82\%), PIP joint synovitis in 23 digits $(68 \%)$ and DIP joint synovitis in 17 digits (50\%). Extensor paratenonitis was observed in 21 digits $(62 \%)$ and flexor tenosynovitis in 20 digits $(59 \%)$. Enthesitis of extensor tendons was present in 21 digits $(62 \%)$ and enthesitis of flexor tendon in 5 digits $(15 \%)$. Osteoproliferation was present in 21 digits (62\%). The frequency of synovitis, tenosynovitis and enthesitis did not differ significantly between tender $(n=25)$ and non-tender $(n=9)$ dactylitis. A significant association between tenderness and the presence of power Doppler $\left(\chi^{2}: 17.9\right.$, $p<0.01)$ and of edema in the soft tissue was observed $\left(x^{2}: 11.5, p<0.01\right)$.

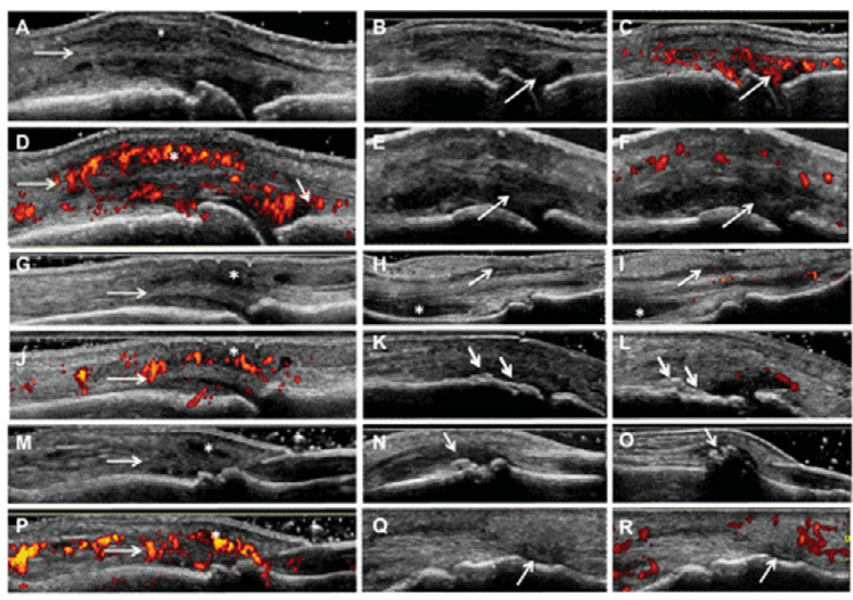

Figure. Ultrasound lesions in dactvlitis. Longitudinal scans of digital extensor tendons in GS (AG,M) and in PD (D, J, P) US showed extensive peritendinous inflammation. Note the tendor thickenim (AGM the loss of fbrillar pattern (AG) and hypochorenicity (A $M$ of the extenso thich at the synovio-entheaseal complex at the DIP joint (MP) (arrows in panels A,D,G,J,MP indicate digital extensor tendon and asterisks indicate anechoic or hypoechoic material around the dorsal extensor tendon with PD signals). Longitudinal US scans of $M C^{2}$ and $P I P$ joints in $G S(B, E)$ and $P D$ $\mathrm{C}, F)$ mode. PD positive $(C)$ and $P D D$ oppler negative $(F)$ synovitis at the $\mathrm{MCP}$ joint $(B, C)$ and at the $P \mathbb{P}$ joint $(E, F)$. Note the pericapsular $P D$ signals in panel $F$ (arrows in panels $B, C, E, F$ indicate synovial hyperthrophy) with (C) or without (F) PD signals. Longitudinal scans of digital flex or tendor (HI) at the PP joint. Tenosynovitis of flexor digital tendon is depicted in GS $(H)$ and $P D(1)$ mode.

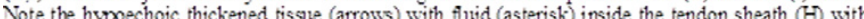
few synovial and intra-tendinous PD signals (I). Osteoproliferation at the PIP joint (KI) and at the $D P$ joint $(N, O)$ depicted in longitudinal scans. Enthe sitis of digital flex or tendon is depicted in GS (Q) and $P D(R)$. The latter finding is rare (arrows indicate enthe sitis).

Conclusions: Dactylitis is a multi-compartment digit disease, and anatomical lesions are more heterogenous than previously described. A significant amount of joint, tendon and entheseal inflammation persists in non-tender dactylitis. Patient-reported tenderness is associated with the presence of edema and/or power Doppler inside the soft tissue.

References:

[1] Bakewell CJ et al. OMERACT Ultrasound Task Force. Ultrasound and magnetic resonance imaging in the evaluation of psoriatic dactylitis: status and perspectives. J Rheumatol. 2013;40:1951-7.

Acknowledgements: We acknowledge S. Aydin and the OMERACT/EULAR working group for fruitful discussions.

Disclosure of Interest: None declared

DOI: 10.1136/annrheumdis-2017-eular.2283

\section{FRI0634 DO ANTHROPOMETRIC FACTORS INFLUENCE MEDIAN NERVE SIZE IN HEALTHY INDIVIDUALS? AN ULTRASOUND AND VOLUMETRY STUDY}

A. O'Neill, P. Gardiner, D. McCormick, E. Savage, D. Small. Rheumatology, Western Health and Social Care Trust, Londonderry, United Kingdom

Background: Some (but not all) ultrasound studies of median nerve dimensions at the wrist have suggested that anthropometric factors such as height and weight are among the possible sources of variability in the normal population but this has not been systematically studied ${ }^{1}$.

Objectives: The purpose of our study was to establish if hand and wrist volume or other standard anthropomorphic factors influenced median nerve size in normal subjects using standard ultrasonographic techniques. If median nerve size is predictably related to anthropomorphic factors, adjusted normal reference ranges would improve the diagnostic precision of the test for carpal tunnel syndrome.

Methods: We studied 30 healthy subjects: 14 females and 16 males. We measured their height, weight, arm span, finger span and wrist circumference. Ultrasonography of the median nerve was carried out at the entrance to the carpal tunnel and at a second level $2 \mathrm{~cm}$ proximal to the wrist using an Esaote MyLab Class C ultrasound with a $12-18 \mathrm{MHz}$ linear probe. The wrist was marked at the distal wrist crease and $2 \mathrm{~cm}$ proximally. Hand and wrist volume were measured using water displacement volumetry.

Results: The intra-rater reliability of volumetry measurements was excellent for both hand and wrist volumetry with a Cronbach alpha of 0.99 and 0.88 respectively. The intra-rater reliability for median nerve measurements was excellent with a Cronbach alpha of 0.96 . The median nerve dimensions were not significantly different between male and female subjects, nor between the dominant and non-dominant hands. The mean median nerve cross sectional area (CSA) at the distal wrist crease was $10.68 \mathrm{~mm}^{2}$ and at a point $2 \mathrm{~cm}$ proximal to this it was $10.18 \mathrm{~mm}^{2}$. The mean difference in CSA between these two points was $0.5 \mathrm{~mm}^{2}$. The median nerve CSA was not significantly related to height, weight or hand size. It was not correlated with hand or wrist volume. No significant differences were found in relation to hand dominance or gender.

Spearman's correlation between median nerve CSA (distal, right wrist) and anthropometric factors

\begin{tabular}{lcc}
\hline & Rho & $\mathrm{p}$ \\
\hline Height & 0.063 & 0.73 \\
Weight & 0.290 & 0.11 \\
BMI & 0.094 & 0.47 \\
Arm Span & 0.115 & 0.38 \\
Finger Span & 0.024 & 0.85 \\
Wrist Circumference & 0.095 & 0.46 \\
Hand Volume & 0.102 & 0.44 \\
Wrist Volume & 0.244 & 0.19
\end{tabular}

Conclusions: Anthropometric factors including BMI, hand/wrist volume were not found to be closely related to median nerve size in normal individuals.

References:

[1] Cartwright MS et al. Ultrasonographic reference values for assessing the normal median nerve in adults. J Neuroimaging. 2009;19(1):47-51.

Disclosure of Interest: None declared

DOI: 10.1136/annrheumdis-2017-eular.3834

\section{FRI0635 DESCRIPTION OF VIDEOCAPILLAROSCOPY OF THE LABIAL MUCOSA IN COMPARISON WITH NAILFOLD VIDEOCAPILLAROSCOPY IN SCLERODERMA}

A.M. Beron, S. Sapag duran, H. Laborde, G. Medina, M. Garcia Carrasco, G. Nasswetter, D. Dubinsky. Rheumatology, Clinical Hospital "Jose de San Martin", University of Buenos Aires., CABA, Argentina

Background: Capillary bed of labial mucosa may be a potentially useful area for assessing abnormalities on the microcirculation. Videocapilaroscopy of the labial mucosa (LVC) in 12/13 scleroderma (SCL) patients showed capillaries disturbance such as great disorganization and anarchic orientation ${ }^{(1)}$.

Objectives: To describe LVC features in SCL and compare them with healthy controls. (HC). To compare nailfold videocapillaroscopy (NVC) with LVC in HC and SCL patients.

Methods: $\geq 16$ years old patients with SCL (ACR-EULAR 2013) were included consecutively since June to July 2016. Smoking patients were excluded. For the evaluation of the LVC we performed a general displaying and then we studied following areas: a-central ( $1 \mathrm{~cm}$ from the frenulum), b-lateral right and left (both 2 $\mathrm{cm}$ from the frenulum) and c-labial border. For evaluation of the NVC, 200x images were obtained from 2nd to 4th fingers both hands and was classified according Cutolo in early, active, late patterns ${ }^{(2)}$. For assesment through LVC: photographs (200x) for each subject were taken. We define quantitative characteristics as means of: capillary diameter, length and density. The average of these parameters was obtained from the evaluation of capillaries included in $1 \mathrm{~mm}^{2}$ of at least 2 continuous images. In addition, the visibility of the subpapillary venous plexus, architecture, dilatation of capillaries, presence of megacapillaries and avascular areas were qualitatively evaluated (photo 1). Each image was reviewed by two experienced observers. We conducted the same process describe above for each healthy control subject.

Results: Twenty two patients were included (18 female, age $47 \pm 11$ ys) with SCL (limited 17, diffuse 5); NVC pattern: early 8, active 11, late 3 . Healthy subjects 12 (11 female, age: $34 \pm 10$ years).

Average capillary density: $17 \pm 4.13(13-26)$ in SCL vs $21 \pm 3.65(16-28)$ in HC (p 0.00).

Average capillary diameter: $43 \pm 13.54$ (17-83) SCL vs $19 \pm 1.67 \mu \mathrm{m}(16-21) \mathrm{HC}$ (p 0.02).

Mean capillary length: $185 \pm 34 \mu \mathrm{m}(125-248)$ SCL vs $237 \pm 42 \mu \mathrm{m}(178-313) \mathrm{HC}$ (p 0.00).

In 22 SCL patients were observed dilated capillaries in 20 (90\%), megacapillaries $(\geq 70 \mu \mathrm{m})$ in $10(45 \%)$ and $7(32 \%)$ showed disturbed architecture with lost areas of the normal $U$-shape and changes in the parallelism of the main axis of the 
capillaries. About subpapillary venous plexus in SCL: 7 (32\%) were prominent and only one had capillary hemorrhage. In contrast, none of the controls presented these alterations. ( $p 0.0$ ).

According to the 3 NVC patterns the following averages were observed in the LVC:

Early Pattern (n: 8): diameter 36.93 $\pm 10.84 \mu \mathrm{m}$; Long $191.21 \pm 47.58 \mu \mathrm{m}$; Density $18.12 \pm 5.33 \mu \mathrm{m}$.

Active Pattern (n: 11): diameter 48.80 $\pm 14.80 \mu \mathrm{m}$; Length 184.11 $\pm 29.91 \mu \mathrm{m}$; Density $16.72 \pm 3.25 \mu \mathrm{m}$.

Late Pattern ( $\mathrm{n}: 3$ ): diameter $36.27 \pm 10$; Long $178.03 \pm 28.8 \mu \mathrm{m}$, density $19.33 \pm 4.16$ $\mu \mathrm{m}$.

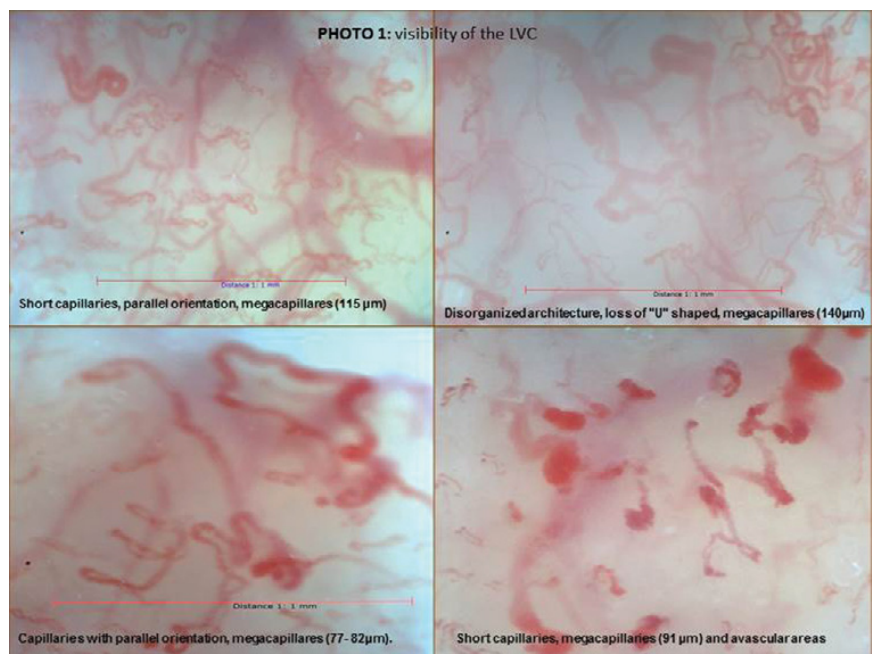

Conclusions: We found that LVC in SCL patients show significant microvascular changes with respect to $\mathrm{HC}$. In addition, all NVC patterns described in SCL showed similar alterations in LVC. Therefore, LVC could be a complementary or alternative method to NVC since it is easily accessible, has good visibility and is not influenced by local mechanical or chemical stimuli that can affect the nail bed. References:

[1] W Grassi et al. Ann Rheum Dis 1993; 52 (8): 564-569.

[2] M. Cutolo et all. Rheumatology 2006; 45: 43-46.

Disclosure of Interest: None declared

DOI: 10.1136/annrheumdis-2017-eular.4329

\section{FRI0636 ENDOTHELIAL DYSFUNCTION IN SYSTEMIC LUPUS ERYTHEMATOSUS - A CASE-CONTROL STUDY AND AN UPDATED META-ANALYSIS AND META-REGRESSION}

A. Mak ${ }^{1}$, N.Y. Kow ${ }^{1}$, H. Schwarz ${ }^{2}$, L.L. Gong ${ }^{3}$, S.H. Tay ${ }^{1}$, L.H. Ling ${ }^{3}$ ${ }^{1}$ Medicine; ${ }^{2}$ Physiology; ${ }^{3}$ Cardiology, National University of Singapore, Singapore, Singapore

Background: Endothelium-dependent flow-mediated dilation (ED-FMD), a biophysical marker of endothelial dysfunction, is apparently impaired in patients with systemic lupus erythematosus (SLE). However, such observation is inconsistent because of the lack of standardization of the methodology of ED-FMD measurement and inclusion of patients with different comorbidities amongst different studies.

Objectives: Firstly, we evaluated if ED-FMD is indeed impaired in SLE patients naïve of cardiovascular disease and its traditional risk factors. Secondly, we aimed to determine if the putative contribution of SLE to endothelial dysfunction is in fact confounded by demographic-, disease- and treatment-related factors.

Methods: We assessed and compared the brachial artery ED-FMD (baED-FMD) using the Prosound Alpha-10 ultrasound system ${ }^{\circledR}$ between SLE patients without cardiovascular disease and cardiovascular risk factors and healthy controls $(\mathrm{HC})$ matched for age, gender and body mass index (BMI). Exclusions were pregnancy, a history hypertension, diabetes mellitus, chronic kidney disease, cardiovascular and cerebrovascular diseases, and statin therapy. SLE-related disease activity and organ damage in the SLE patients were assessed using SELENA-SLEDAI and SLICC/ACR DI, respectively. With inclusion of our own data from this case-control study, we performed a comprehensive meta-analysis of case-control studies which compared baED-FMD between SLE patients and HC by determining the effect size of baED-FMD as standard mean difference (SMD). Demographic and clinical factors associated with the effect size were explored by mixed-model meta-regression.

Results: Seventy one SLE patients and $71 \mathrm{HC}$ were studied, and there were 6 men in each group. The mean $\pm S D$ age and BMI of SLE patients and HC were $39.21 \pm 13.4$ and $40.37 \pm 12.9$ years, and $22.54 \pm 5.1$ and $22.86 \pm 4.2 \mathrm{~kg} / \mathrm{m}^{2}$, respectively. In SLE patients, the mean $\pm S D$ daily prednisolone dose, SELENASLEDAI and SLICC/ACR DI were $13.43 \pm 14.4 \mathrm{mg}, 6.52 \pm 5.4$ and $0.17 \pm 0.4$, respectively. SLE patients had significantly lower baED-FMD than $\mathrm{HC}(3.72 \pm 2.8 \%$ vs $4.63 \pm 3.1 \%, p=0.032)$. In the SLE group, no association between baED-FMD and age, gender, BMI, blood pressure, duration of SLE, serum C3, C4 and antidsDNA levels, SELENA-SLEDAI, SLICC/ACR DI and daily prednisolone dose was found. Similarly, no association was noted between baED-FMD and age, gender and $\mathrm{BMI}$ in the $\mathrm{HC}$ group. Meta-analysis of 25 case-control studies involving 1,313 SLE patients and $1,012 \mathrm{HC}$ with the random effects model revealed significantly lower baED-FMD in SLE patients compared to HC (SMD -1.077, $\mathrm{p}<0.001$ ) (see Fig for forest plot). The presence of diabetes mellitus $(p=0.04747)$, higher diastolic blood pressure $(p=0.04419)$ and renal involvement $(p=0.02721)$ were associated with more discrepant baED-FMD between both groups.

stcocrame

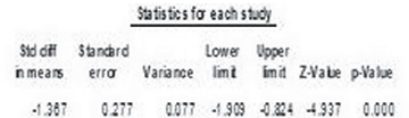

$\begin{array}{llllllll}\text { themad.2009 } & -1.367 & 0.277 & 0077 & 1.909 & 0.324 & +937 & 0.000\end{array}$

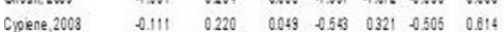
$\begin{array}{lllllllll}\text { Dhang. 2009 } & 0.858 & 0.198 & 0.035 & -1.027 & 0.289 & 3.493 & 0.000\end{array}$ $\begin{array}{llllllll}\text { Drang, 2009 } & 0.868 & 0.188 & 0.035 & -1.027 & 0.289 & 3.493 & 0.000 \\ \text { Vativelso, 2008 } & 0.885 & 0.307 & 0.094 & -1.487 & 0.283 & -2.880 & 0.004\end{array}$ $\begin{array}{lllllllll}\text { Sienungsson, } 2008 & 0.282 & 0.279 & 0.078 & 0.266 & 0.228 & 1.010 & 0.312\end{array}$ $\begin{array}{lllllllll}\text { Fiper.2007 } & 0.543 & 0.275 & 0.076 & -1008 & 0.003 & -1.971 & 0.049\end{array}$

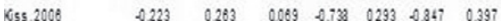

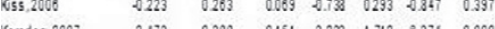

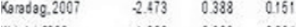
Whight $2006 \quad+1.000 \quad 0.300 \quad 0.094 \quad+80000.000 \quad 3.368 \quad 0.001$ Raggopalam,2004 $\quad 0.800 \quad 0.224 \quad 0.050 \quad+239 \quad 0.381 \quad 3.569 \quad 0.000$ $\begin{array}{llllllll}\text { Lima.2002 } \quad+307 & 0.228 & 0.051 & \$ .761 & -0.384 & 5.373 & 0.000\end{array}$ Hom

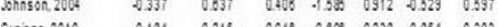

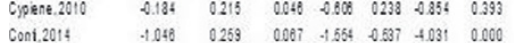
$\begin{array}{lllllllll}\text { Bassolou.2018 } & 0.302 & 0.114 & 0.013 & 0.099 & 0545 & 2.833 & 0.005\end{array}$

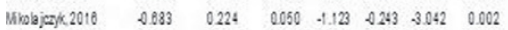
$\begin{array}{lllllllll}50 m e s .2012 & \$ .375 & 0.193 & 0.037 & \$ .753 & 0.004 & .9 .938 & 0.053\end{array}$

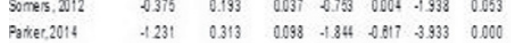
her: 2009

อ.8.

Snoer, 2015

Pramanik, 2011

Vake, 2013

Mak (presentstoy)

$\begin{array}{lllllll}0.000 & 0.281 & 0.068 & 0.511 & 0511 & 0.000 & 1.000 \\ 4939 & 0.484 & 0.215 & 5893 & 4031 & .0865 & 0.000\end{array}$

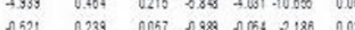

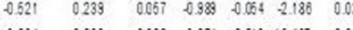

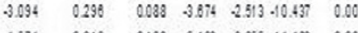

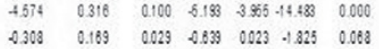

$\begin{array}{llllll}.077 \quad 0214 \quad 0048 & 1.497 & 0.057 & 8.025 & 0.000\end{array}$

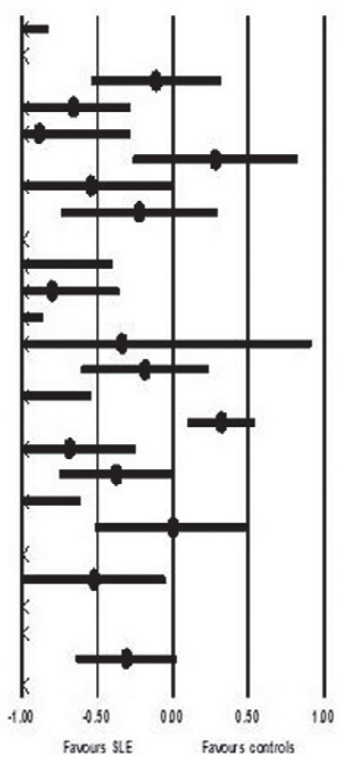

Conclusions: SLE patients naïve of cardiovascular disease and its traditional risk factors have impaired endothelial function. While SLE-related disease activity and organ damage are not apparently related to endothelial dysfunction, the presence of diabetes mellitus, renal disease and diastolic hypertension are potential contributors to endothelial dysfunction in SLE patients.

Disclosure of Interest: None declared

DOI: 10.1136/annrheumdis-2017-eular.4350

\section{FRI0637 DIAGNOSTIC PERFORMANCE OF THE NEW OMERACT CRITERIA FOR CPPD IDENTIFICATION BY US: CORRELATION WITH SYNOVIAL FLUID ANALYSIS}

A. Adinolfi ${ }^{1}$, V. Picerno ${ }^{1}$, A. Scanu ${ }^{2}$, C. Toscano ${ }^{1}$, C.A. Scirè ${ }^{3}$, G. Carrara ${ }^{4}$, B. Frediani ${ }^{1}$, A. lagnocco ${ }^{5}$, N. Damjanov ${ }^{6}$, L. Punzi ${ }^{2}$, G. Filippou ${ }^{1}$. ${ }^{1}$ University of Siena, Siena; ${ }^{2}$ University of Padova, Padova; ${ }^{3}$ University of Ferrara, Ferrara; ${ }^{4}$ SIR-Epidemiology Unit, Milan; ${ }^{5}$ University of Turin, Turin, Italy; ${ }^{6}$ University of Belgrade, Belgrade, Serbia

Background: Ultrasonography (US) has demonstrated to be an accurate tool for the diagnosis of calcium pyrophosphate deposition disease (CPPD) (1). Recently, the OMERACT "US in CPPD" subtask force, has created new definitions for CPPD identification by US, that demonstrated to be reliable at the knee joint, bridging a gap afflicting the old definitions (2). On the other hand, synovial fluid analysis (SFA) is considered to be an accurate and valid method for diagnosing CPPD.

Objectives: The aim of this study wasto evaluate the association between US, using for the first time the new OMERACT US criteria for CPPD, and SFA findings for identifying patients affected by CPPD.

Methods: We enrolled all the consecutive patients, aged more than 60 years old, referred to our outpatient clinic from September 2016 to December 2016, for knee pain and that presented knee effusion of any grade. Patients with suspected chronic inflammatory conditions were excluded.

All the subjects underwent an US exam (EsaoteMyLab 70) of the clinically involved knee, performed by an expert sonographer that applied the new OMERACT criteria for the diagnosis of CPPD at the fibrocartilage (menisci) and hyaline cartilage of the affected knee (2).

Subsequently, a US-guided arthrocentesis was performed, and the synovial fluid was collected and analyzed by a compensated polarized light microscopy (AxioLab A.1 [Zeiss]) by an expert observer in order to assess the presence of CPP crystals. Both observers were blinded to clinical and to each other findings. The Chi-squared test was used to correlate the US and SFA findings.

Results: 49 patients ( 28 women) were enrolled in the study, with a mean age of $70,29$ yo (SD $\pm 10,93)$. 28 subjects were affected by CPPD at SFA and 26 patients 